\title{
Magnetic field induced assembling of nanoparticles in ferrofluidic liquid thin films based on $\mathrm{Ni}_{x} \mathrm{Fe}_{1-x} \mathrm{Fe}_{2} \mathrm{O}_{4}$
}

\author{
V S ABRAHAM ${ }^{\dagger}$, S SWAPNA NAIR, S RAJESH ${ }^{\#}$, U S SAJEEV and \\ M R ANANTHARAMAN* \\ Department of Physics, 'International School of Photonics, Cochin University of Science and Technology, \\ Cochin 682 022, India \\ \#Biophysics and Microscopy Group, University of Amsterdam, The Netherlands
}

MS received 19 July 2003

\begin{abstract}
Ultra fine precursors for ferrofluid synthesis, belonging to the series, $\mathrm{Ni}_{x} \mathrm{Fe}_{1-x} \mathrm{Fe}_{2} \mathrm{O}_{4}$ (where ' $x$ ' varies from ' 0 ' to ' 0.6 ' in steps of 0.1 ), were synthesized. Ferrofluids based on these fine particles were prepared with oleic acid as surfactant and kerosene as carrier. Ferrofluidic thin films were made on glass substrates and magnetic field induced laser transmission was studied. The pattern exhibited by the films under the influence of a magnetic field was observed with the help of a CCD camera. The analysis of results confirms the chain formation of particles in the presence of an applied magnetic field and their saturation at higher applied fields.
\end{abstract}

Keywords. Ferrofluids; nickel ferrite; field induced assembling; ultra fine particles.

\section{Introduction}

Ferrofluids are stable suspensions of nanomagnetic particles in a suitable base fluid. They are of great research interest because of their numerous physical, engineering and medical applications (Rosenweig 1985; Berkovsky et al 1993; Vasile and Aurora 1994). These smart fluids show many interesting magneto-optical properties. Some of the engineering applications of these rheological fluids are in making rotary seals, pressure sensors and loud speakers. They are also widely used in bio-medical fields especially in cancer therapy (Vasile and Aurora 1994).

The optical and magnetic properties of these rheological fluids have been widely studied (Davies and Llewellian 1979; Luo et al 1991; Do et al 1999; Ishi 1998). Magnetic field induced structural anisotropy gives rise to many special magneto-optical properties like field induced optical birefringence, linear and circular dichroism, Faraday rotation and ellipticity (Chikazumi et al 1987). They also exhibit magnetic field independent properties like zero field birefringence (Hong 1999). The effect of a magnetic field on these thin films will have a profound influence on the birefringent properties of these fluid films. If an external magnetic field is applied parallel to the ferrofluidic thin films, they form tiny clusters that grow in size in increasing fields, which contributes to optical birefringence.

\footnotetext{
*Author for correspondence
}

The cold co-precipitation technique is an excellent method for the preparation of powder precursors for ferrofluids. Many of the mixed ferrites can be prepared by cold co-precipitation technique and they can be further ground by HEBM for reducing the particle size further and they can serve as precursors for ferrofluid preparation. It is expected that cold co-precipitation of a series similar to $\mathrm{Ni}_{x} \mathrm{Fe}_{1-x} \mathrm{Fe}_{2} \mathrm{O}_{4}$ will result in fine particles and further high energy ball milling (HEBM) will enable to prepare ultra fine particles with various particle dimensions.

Nickel ferrite crystallizes in an inverse spinel configuration with nickel having exclusive octahedral site preference (Smit and Wijn 1959; Kittel 1971). The nickel ferrite nanoparticles show many distinctive magnetic properties. They exhibit superparamagnetism, characteristic of a nanoferrite having the size distribution of a few nanometers (Eberbeck and Ahlers 1999; Spinu et al 2000). In nickel ferrite nano particles, due to a noncollinear spin structure, there is a strong reduction in the magnetization value compared with the bulk value. It is reported that due to the spin-glass-like layer wrapped around a uniformly magnetized core, hysteresis loop often shows open loop behaviour even at very high applied fields (Hasmonay and Depeyrot 2000).

The series, $\mathrm{Ni}_{x} \mathrm{Fe}_{1-x} \mathrm{Fe}_{2} \mathrm{O}_{4}$, with increasing ' $x$ ' content in steps of $0 \cdot 1$ will exhibit a behaviour different from that shown by the parent $\mathrm{Fe}_{3} \mathrm{O}_{4}$ in a serial manner due to the increasing ' $\mathrm{Ni}$ ' content. Moreover, the variation of optical behaviour with ' $x$ ' value can be studied. The optical and 
magnetic properties can be compared by the investigation of magnetic field induced laser transmission through these ferrofluid films. Due to these peculiar properties, the nickel series is chosen for the preparation of the ferrofluids.

A survey of the literature reveals an absence of a systematic study on the optical properties of ferrofluidic thin films prepared from a series similar to $\mathrm{Ni}_{x} \mathrm{Fe}_{1-x} \mathrm{Fe}_{2} \mathrm{O}_{4}$. Moreover, a correlation of the observed optical properties of these ferrofluidic thin films with the magnetic properties of the precursors will throw a deeper insight as regard to cluster formation under the influence of an external magnetic field.

In the present investigation, powder samples belonging to the series, $\mathrm{Ni}_{x} \mathrm{Fe}_{1-x} \mathrm{Fe}_{2} \mathrm{O}_{4}$, where ' $x$ ' varies from 0 to 0.6 in steps of $0 \cdot 1$, were synthesized by cold co-precipitation technique. These powders were then subjected to HEBM with oleic acid and finally with kerosene to prepare ferrofluids. The structural properties of these precursor materials were studied. Ferrofluid liquid thin films were then prepared and magnetic field induced laser transmission through these ferrofluid liquid thin films was studied for different compositions. Attempts were made to correlate their magnetic and the corresponding optical properties.

\section{Experimental}

\subsection{Preparation of magnetic fine particles}

Fine particles of nickel iron ferrite, which are the precursor material for the preparation of the ferrofluids, were synthesized by cold co-precipitation. For this, $\mathrm{NiSO}_{4} \cdot 6 \mathrm{H}_{2} \mathrm{O}$, $\mathrm{FeSO}_{4} \cdot 7 \mathrm{H}_{2} \mathrm{O}$ and $\mathrm{FeCl}_{3}$ (all AR grade chemicals) in the molar ratio of $x \mathrm{M},(1-x) \mathrm{M}$, and $2 \mathrm{M}$ were employed (Morosov et al 1987; Sutharia et al 2001; Wu et al 2001).

\section{$2.2 X$-ray diffraction studies}

$\mathrm{X}$-ray diffraction of the samples were recorded in an $\mathrm{X}$ ray diffractometer (Rigaku Dmax-C) using $\mathrm{Cu} \mathrm{K} \alpha$ radiation $(\lambda=1.5418 \AA)$ (Malini et al 2001). Lattice parameter (a) was calculated assuming cubic symmetry (Cullity 1978) and planes were identified by matching interplanar spacing values $(d)$ with standard tables (JCPDS-ICDD C, 1990). The average particle sizes of these powder samples were estimated by employing Debye Scherrer's formula

$$
D=\frac{0 \cdot 9 \lambda}{\beta \cos \theta},
$$

where $\lambda$ = wavelength of X-ray used in $\AA$, $\beta=$ FWHM in radians of the XRD peak with highest intensity, $D=$ par- ticle diameter in $\AA$, $\theta=$ the angle of diffraction in radians.

\subsection{Reduction of particle size}

Particle size was reduced by high energy ball milling. For the preparation of ultra fine particles, 'Fritsch pulverisette 7 planetary micro mill' was employed. In this, 800 rotations per min can be achieved and hence the momentum imparted to the particles will be very high.

\subsection{Ferrofluid preparation}

Ferrofluids were prepared by milling the powder samples obtained by cold co-precipitation with the surfactant oleic acid. Oleic acid provides the necessary steric repulsion by its polar ends, thus preventing the agglomeration of the fine particles. Finally these samples were milled with kerosene for around $3 \mathrm{~h}$ to obtain a uniform stable dispersion with a reduced particle size. The samples were centrifuged well to remove the sedimented particles and placed in an ultrasonic vibrator to enhance Brownian motion, which provides stability to the ferrofluid. Oleic acid, kerosene and nickel ferrite particles were optimally employed to ensure good quality ferrofluid. Ferrofluid thus prepared exhibited typical characteristics like spiking, which is a sure test for the formation of ferrofluids.

\subsection{Ferrofluid film preparation}

Liquid thin films of these ferrofluids were then made by sandwiching and encapsulating $2 \mathrm{~mm}^{3}$ of ferrofluid between two optically smooth and ultrasonically cleaned glass slides. The thickness of these fluid films was of the order of a few thousands of angstroms.

\subsection{Experimental set up for field induced laser transmission through ferrofluid film}

The ferrofluidic film was suspended between the poles of a powerful electromagnet whose magnetic field strength is precalibrated. The electromagnet can go up to a field of 7500 Gauss with water circulation for cooling. The film sample was then irradiated with a diode laser having a power of $3 \mathrm{~mW}$. The wavelength was in the range 630 $670 \mathrm{~nm}$. The fluid film was aligned in such a way that the applied magnetic field is perfectly parallel to the plane of the aligned film. The laser beam was focused normally on to the film sample and the light transmitted by the ferrofluid film sample was caught on a screen, placed at a distance of $0.5 \mathrm{~m}$ from the film. The entire experimental set up is as shown in figure 1 . The power of the transmitted beam is measured using a laser power meter (OPHIRPD 200 having $0.001 \mathrm{~mW}$ accuracy) in the gradually in- 
creasing magnetic field. The exact field was measured each time with a digital Gaussmeter (Model DGM-102 with maximum field reading capacity up to 20,000 G). The magnetic field was calibrated in terms of the optical output intensity for all these fluid film samples belonging to the series, $\mathrm{Ni}_{x} \mathrm{Fe}_{1-x} \mathrm{Fe}_{2} \mathrm{O}_{4}$.

\subsection{Imaging of chain formation}

The thin ferrofluid film was kept in different magnetic fields and allowed to dry in the respective applied fields. These dried ferrofluid films were viewed with the help of a CCD camera (Model No. G P KR 222) and an optical microscope and the pattern obtained was imaged on a colour video monitor and was recorded photographically.

\section{Results and discussion}

The analysis of the X-ray diffraction results indicates that the prepared compounds crystallize in a spinel phase. The particle size evaluation employing Debye Scherrer's formula suggests that they lie in the range 50-95 $\AA$ for different samples with varying nickel contents. It must be noted that the phase formation is complete in the cold precipitated state itself. The particle size and lattice parameter variation for all the series is given in table 1 . The lattice parameter variation is plotted with composition $(x)$, which shows a linear decrease of lattice parameter with composition for the series, $\mathrm{Ni}_{x} \mathrm{Fe}_{1-x} \mathrm{Fe}_{2} \mathrm{O}_{4}$ (figure 2). This is in accordance with the Vegard's law (Smit and Wijn 1959). According to Vegard's law, the lattice parameter of a solid solution is directly proportional to the atomic percentage of the solute present in it. Here in the series, $\mathrm{Ni}_{x} \mathrm{Fe}_{1-x} \mathrm{Fe}_{2} \mathrm{O}_{4}$, as ' $x$ ' increases, the atomic percent-

3

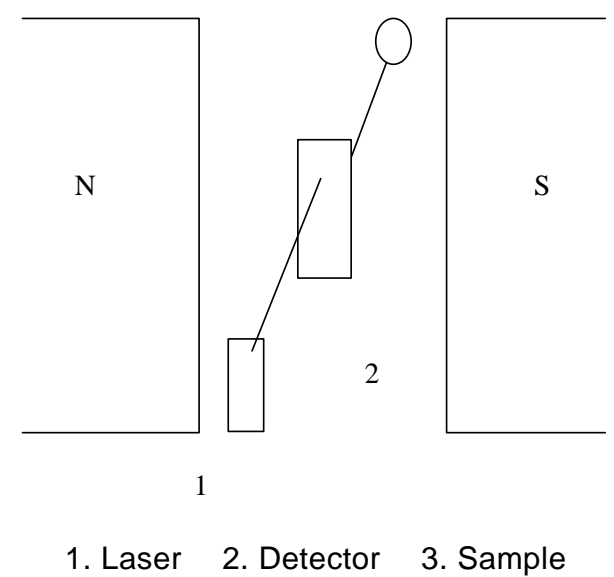

Figure 1. Experimental set up for field induced laser transmission through ferrofluid thin film (Normal incidence and detection with respect to the sample). age 'Fe' decreases which reduces the lattice parameter value. The evaluation of the particle size is important at various states of milling in ferrofluid preparation as it controls many of the peculiar properties exhibited by these fluids and it has been shown that particles below a critical size of $100 \AA$ will be single domain in nature and have superparamagnetic characteristics. Moreover, these particles will undergo Brownian motion, thereby increasing their stability, if dispersed in a carrier liquid only under this critical size value.

As the ferrofluid liquid film restricts the motion of particles in a plane, application of a magnetic field increases structural anisotropy of these particles and agglomeration starts at a very low magnetic field and the particles self assemble themselves in presence of applied magnetic field, yielding long chains and this process gets saturated at higher fields giving rise to a two dimensional quasi continuous grating (Hong 1999; Horng et al 2001). Thin long chains of varying thickness are formed leading to optical anisotropy, which contributes to optical birefringence.

This is an example of field induced assembling of nanomaterials. The chain formation is confirmed by photographs taken in different applied fields with the help of a ccd camera and are shown in figures $3 \mathrm{a}-\mathrm{d}$. The chain formation saturates in higher fields. It is observed that

Table 1. Composition vs particle size for $\mathrm{Ni}_{x} \mathrm{Fe}_{1-x} \mathrm{Fe}_{2} \mathrm{O}_{4}$.

\begin{tabular}{lc}
\hline Composition $(x)$ & Particle size $(\AA)$ \\
\hline $0 \cdot 1$ & 94 \\
$0 \cdot 2$ & 82 \\
$0 \cdot 3$ & 59 \\
$0 \cdot 4$ & 52 \\
$0 \cdot 6$ & 77 \\
\hline
\end{tabular}

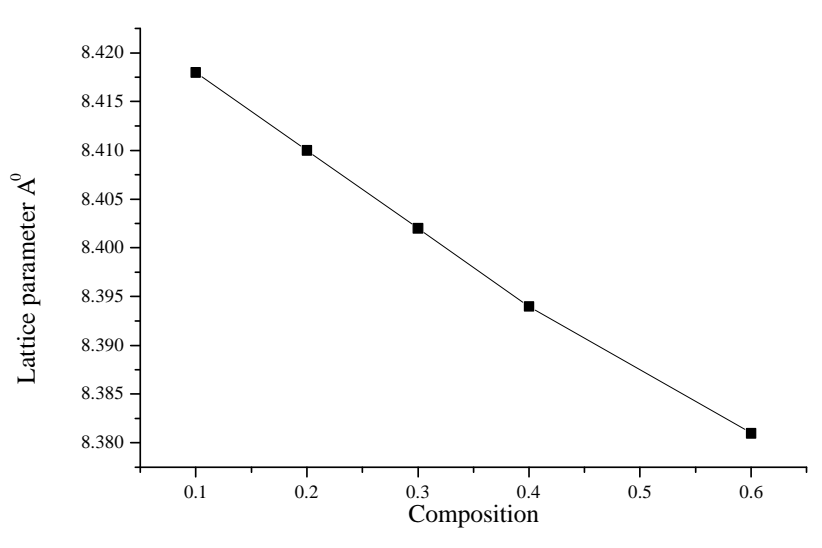

Lattice parameter vs composition

Figure 2. Lattice parameter $(\AA)$ vs composition ' $x$ ' in the series, $\mathrm{Ni}_{x} \mathrm{Fe}_{1-x} \mathrm{Fe}_{2} \mathrm{O}_{4}$. 
(a)

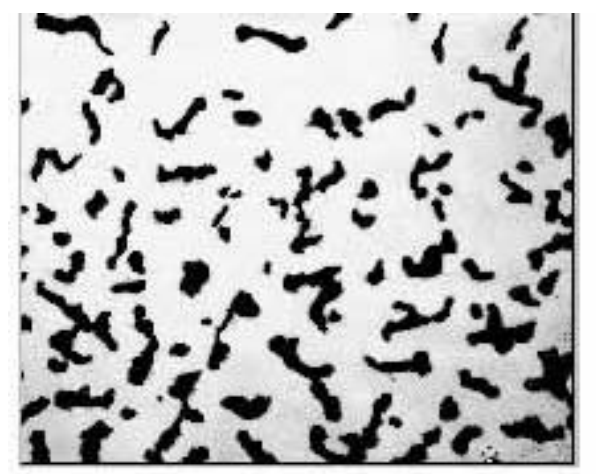

(c)

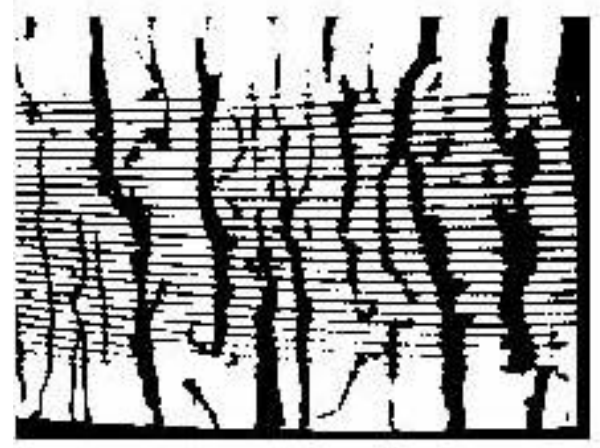

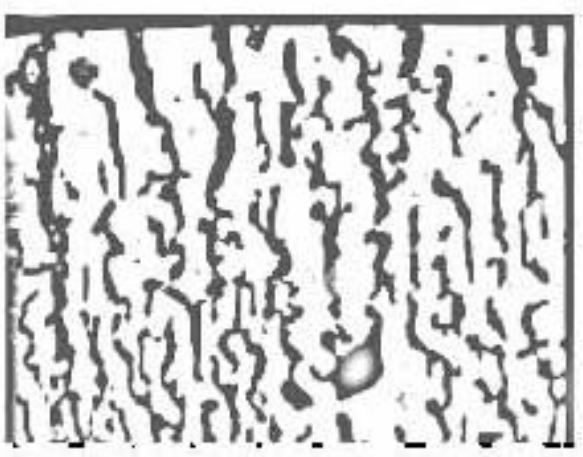

(b)

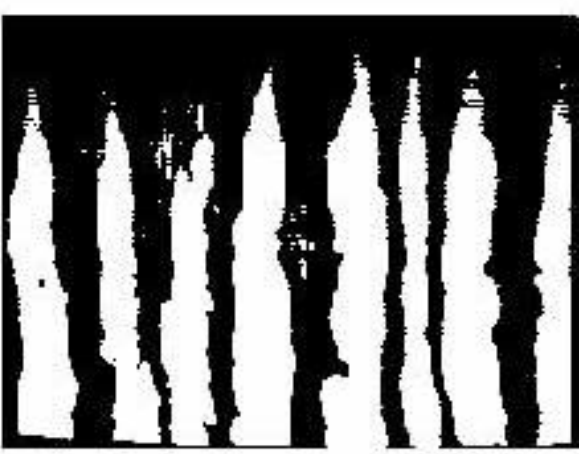

(d)

Figure 3. Photographs showing the chain formation ferrofluid films taken using ccd camera in different magnetic field values (a. $1390 \mathrm{G}$, b. $2210 \mathrm{G}$, c. $2690 \mathrm{G}$ and d. $3980 \mathrm{G}$ ).

the diffracted light intensity increases with applied magnetic field. This is due to the increase in cluster formation in the ferrofluidic thin film with increasing magnetic field. This can diminish the central spot intensity. As the field increases the central spot intensity decreases in a linear manner and then attains a steady value indicating saturation of the cluster formation.

The optical properties of ferrofluids are dictated by the change in refractive index, $\Delta n$, which can be related to the orientational tensor component parallel to the anisotropy axis, $S_{\mathrm{zz}}$ as

$$
\begin{aligned}
& \Delta n=B \varepsilon S_{\mathrm{zz}}, \\
& B=\left(1 / 2 n_{0}\right)\left(\chi_{\|}-\chi_{\perp}\right),
\end{aligned}
$$

where $n_{0}$ is the refractive index of the carrier, $\varepsilon$ the dielectric permittivity, and $S_{\mathrm{zz}}$ the orientational tensor and $\chi_{\|}$and $\chi_{\perp}$ are the effective susceptibility of the magnetic particles parallel and perpendicular to the anisotropy axis. So evaluating refractive index changes using this relation, only the Zeeman energy term $\left(E_{\mathrm{z}}=-\mu H \cos \theta\right)$ is included in calculation and so in the weak interaction approximation (Wu and Yao 1999; Horng et al 2001)

$$
\Delta n / B \varepsilon=3 / 2\left(\left\langle\cos ^{2} \theta\right\rangle-1 / 3\right)=L_{2}(\xi)
$$

where $L_{2}(\xi)$ is the second Langevin function and $\xi=\mu H /$ $k T$.
In the low field limit, $\xi=\mu H / k T \perp 1$ and $\Delta n$ varies linearly with $H^{2}$, thus leading to zero birefringence at zero field (Scholten et al 1980). Similarly it can be concluded that the transmitted intensity at the centre should be diminished linearly with $H^{2}$ in low magnetic field values.

As the magnetic field is increased the particles are aligned and that increases the transparency and more and more clusters are formed (Promislow et al 1995; Wu and Yao 1999). The light gets diffracted by these narrow wires of ferrofluid clusters acting as a quasi continuous grating and a streak of light is obtained as the transmitted ray. This phenomenon of diffraction of light by the ferrofluid thin film can be explained by treating the film in the presence of the applied magnetic field as a system of thin wires with variable thicknesses and spacing in between them. Then different orders of the diffracted beam overlaps and a streak of light is obtained on the screen. This is similar to a diffraction pattern obtained from a thick wire. The diffraction is governed by the relation

$$
2 d \sin \theta=n \lambda
$$

Here, as there are a lot of thin wires of irregular thickness inside the film in the presence of a magnetic field, the situation is similar to the case of ' $\theta$ ', the angle of diffraction becoming very large. This may be one of the reasons for getting a continuous streak of light. There is also a possibility for optical birefringence caused by the aniso- 
tropy created by the application of magnetic field. This is mainly due to the anisotropy in the formation of long chains in the applied magnetic field in addition to the anisotropy induced by the application of a magnetic field in the individual particles.

The normalized intensity vs applied magnetic field is plotted for the ferrofluid liquid thin films corresponding to $x=0 \cdot 1,0 \cdot 2,0 \cdot 3,0 \cdot 4,0 \cdot 6$. They are shown in figures $4-$ 8. The saturating field $\left(H_{\mathrm{s}}\right)$ is determined for each of the composition $(x)$ and are plotted for varying ' $x$ '. They are shown in figure 9. The normalized power decreases in a linear manner with the applied magnetic field in the low magnetic field values for each composition $(x)$. This result can be explained by studying the saturation magnetization values of these ferrofluid film samples.

The saturation magnetization $\left(M_{\mathrm{s}}\right)$ of these compounds can be theoretically calculated by employing Neel's twosublattice model (Smit and Wijn 1959). In this model, it is assumed that the ferrimagnetic properties arise as a result of the interaction of the tetrahedral (A) and octahedral

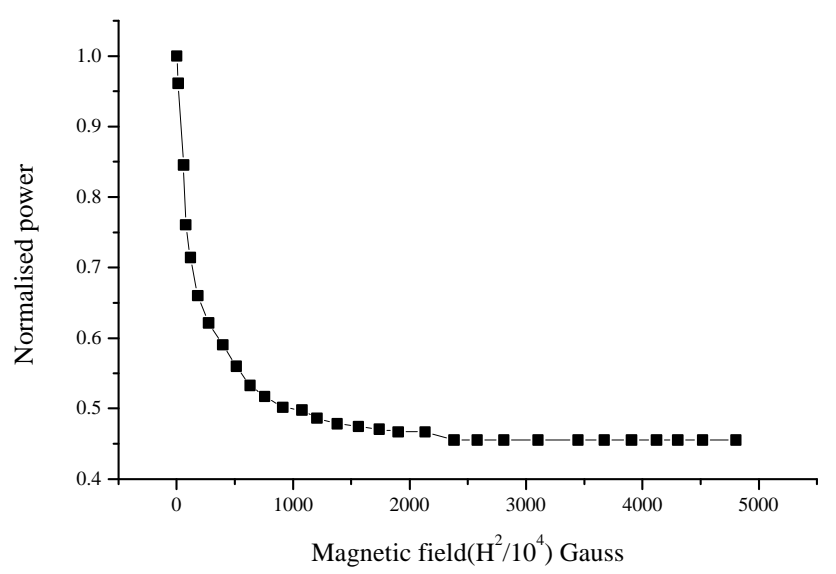

Figure 4. Magnetic field $\left(H^{2} / 10^{4}\right)$ in gauss vs normalized power in $\mathrm{Ni}_{0.1} \mathrm{Fe}_{0.9} \mathrm{Fe}_{2} \mathrm{O}_{4}$.

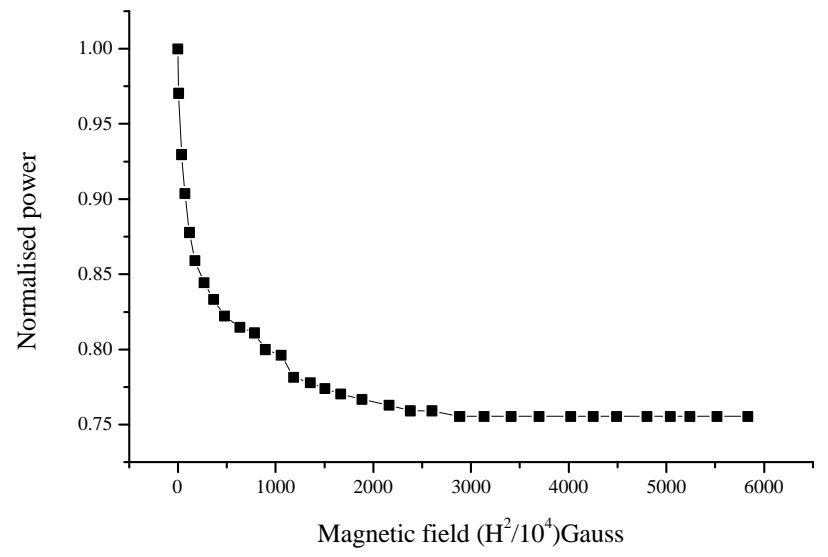

Figure 5. Magnetic field $\left(H^{2} / 10^{4}\right)$ in gauss vs normalized power in $\mathrm{Ni}_{0.2} \mathrm{Fe}_{0.8} \mathrm{Fe}_{2} \mathrm{O}_{4}$.
(B) sublattices. A general cation distribution for the series is assumed and is as follows.

$$
\left[\mathrm{Fe}^{3+}, \mathrm{Fe}_{1-x}^{2+}\right]_{\mathrm{A}}\left[\mathrm{Ni}_{x}, \mathrm{Fe}^{3+}\right]_{\mathrm{B}} \mathrm{O}_{4}^{2-},
$$

where ' $x$ ' varies from $0 \cdot 1,0 \cdot 2,0 \cdot 3,0 \cdot 4$ and $0 \cdot 6$.

The magnetic moment of ferrites is due to the interactions of the moments of the ions in A and B sites and the net magnetic moment value for the bulk sample using the Neel's two sublattice model is given by

$$
\mu=M_{\mathrm{A}}-M_{\mathrm{B}},
$$

where $M_{\mathrm{A}}$ is the total magnetic moment of the A site and $M_{\mathrm{B}}$ that of the $\mathrm{B}$ site. The saturation magnetization in $\mathrm{emu} / \mathrm{g}$ of the samples is calculated by employing the formula

$$
M_{\mathrm{s}}=\frac{5585 \mu}{\text { Mol.wt }}
$$

where $\mu$ is the net magnetic moment in emu of that composition.

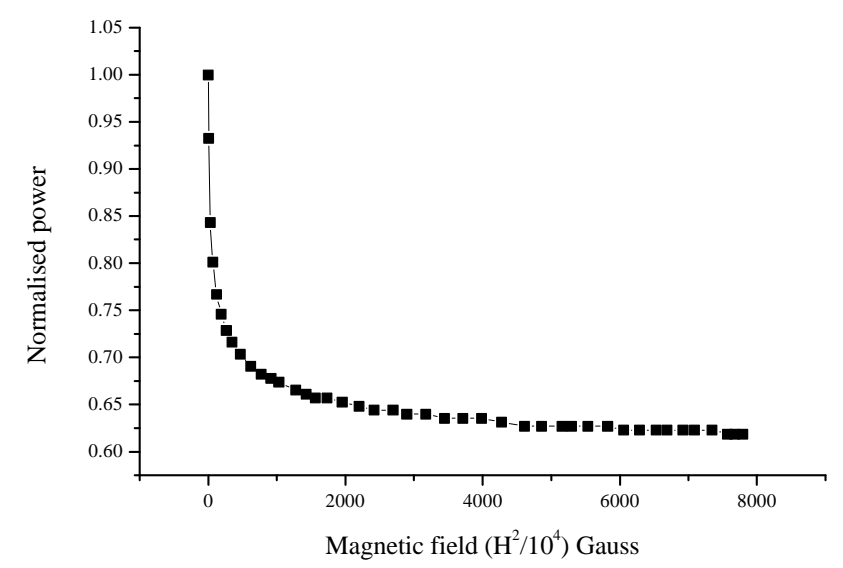

Figure 6. Magnetic field $\left(H^{2} / 10^{4}\right)$ in gauss vs normalized power in $\mathrm{Ni}_{0.3} \mathrm{Fe}_{0.7} \mathrm{Fe}_{2} \mathrm{O}_{4}$.

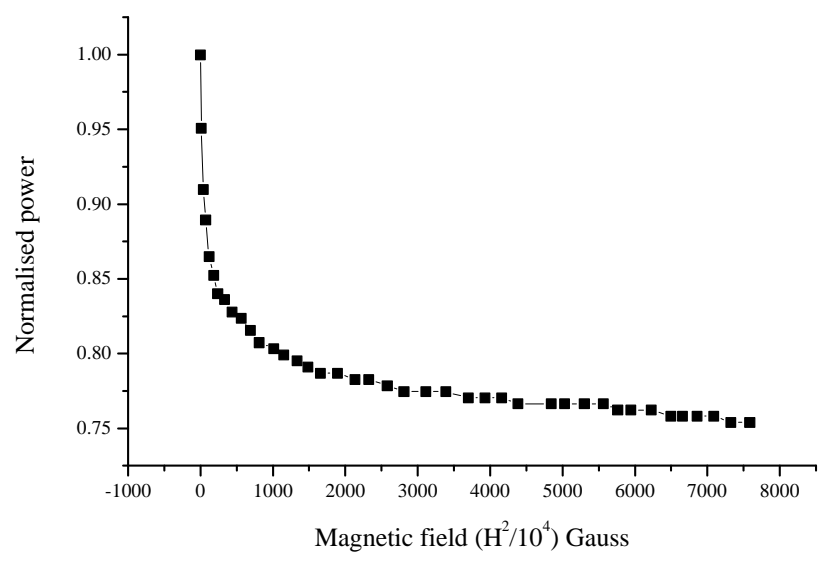

Figure 7. Magnetic field $\left(H^{2} / 10^{4}\right)$ in gauss vs normalized power in $\mathrm{Ni}_{0.4} \mathrm{Fe}_{0.6} \mathrm{Fe}_{2} \mathrm{O}_{4}$. 


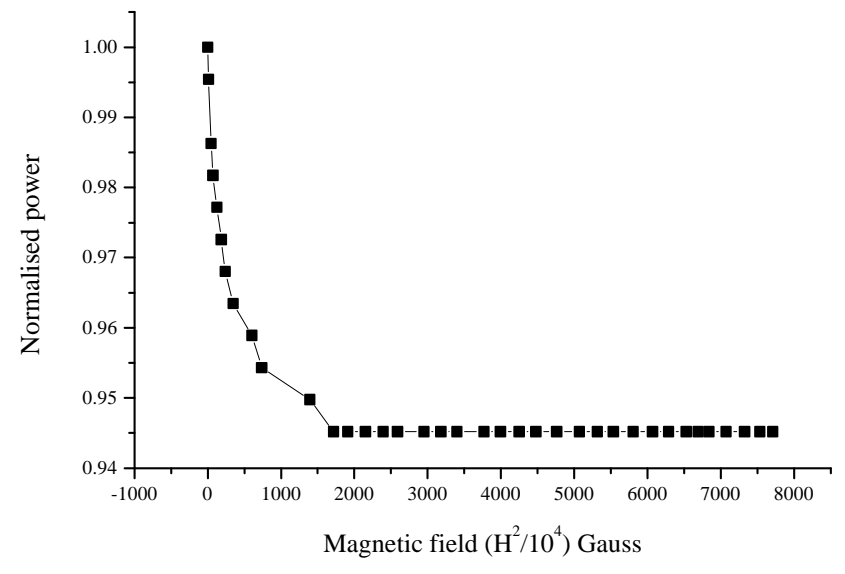

Figure 8. Magnetic field $\left(H^{2} / 10^{4}\right)$ in gauss vs normalized power in $\mathrm{Ni}_{0.6} \mathrm{Fe}_{0.4} \mathrm{Fe}_{2} \mathrm{O}_{4}$.

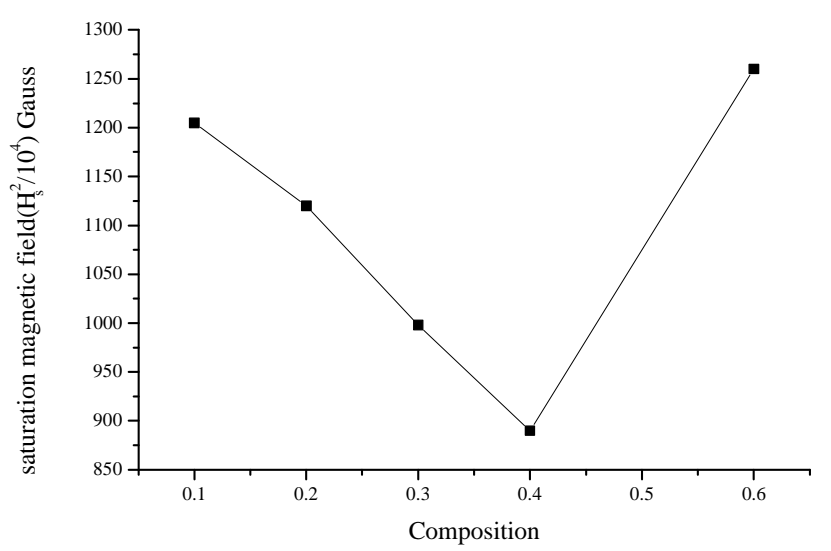

Figure 9. Saturation magnetic field $\left(H^{2} / 10^{4}\right)$ in gauss vs composition ' $x$ ' in $\mathrm{Ni}_{x} \mathrm{Fe}_{1-x} \mathrm{Fe}_{2} \mathrm{O}_{4}$.

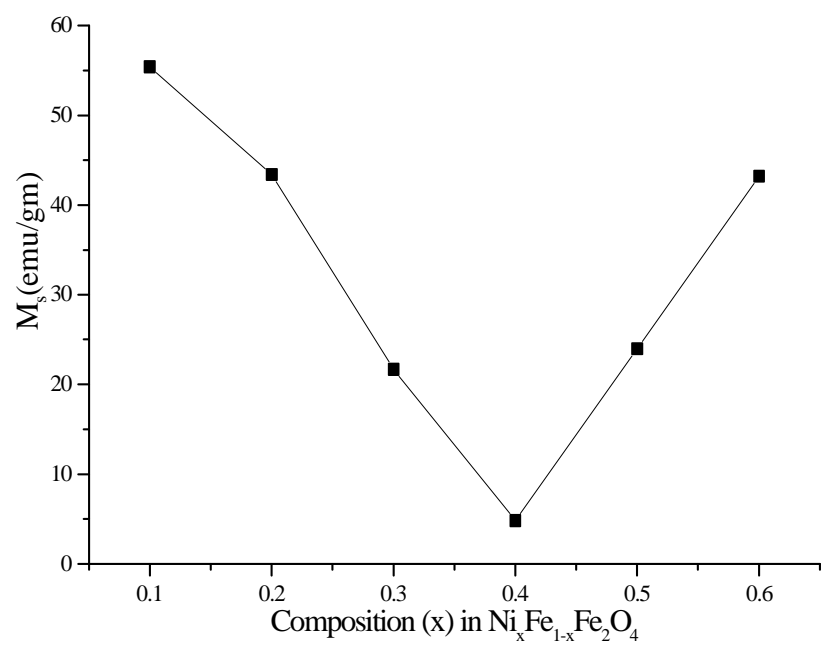

Figure 10. Saturation magnetization, $M_{\mathrm{s}}(\mathrm{emu} / \mathrm{g})$ vs composition ' $x$ ' in $\mathrm{Ni}_{x} \mathrm{Fe}_{1-x} \mathrm{Fe}_{2} \mathrm{O}_{4}$.
This relation is derived as per the above mentioned cation distribution, which has been verified to be valid for the coarser sized $\mathrm{Ni}_{x} \mathrm{Fe}_{1-x} \mathrm{Fe}_{2} \mathrm{O}_{4}$. However, recently they have found a totally different cation distribution in fine $\mathrm{Ni}_{x} \mathrm{Fe}_{1-x} \mathrm{Fe}_{2} \mathrm{O}_{4}$ with respect to their coarser sized counterparts (Anantharaman et al 1998). Nickel may not always occupy the B site in many of these nano particles. It must be noted that the absolute values of ' $M_{\mathrm{s}}$ ' in the fine particle regime can produce deviation from that of the bulk, but the overall variation pattern for various ' $x$ ' will remain the same.

A graph is plotted with calculated saturation magnetization $\left(M_{\mathrm{s}}\right)$ against the composition ' $x$ '. This shows a linear decrease with the ' $x$ ' value in the series, $\mathrm{Ni}_{x} \mathrm{Fe}_{1-x} \mathrm{Fe}_{2} \mathrm{O}_{4}$, up to $x=0.4$ and increases thereafter which is shown in figure 10. This graph shows similar variational pattern for saturation field $\left(H_{\mathrm{s}}\right)$. The variation pattern for $M_{\mathrm{s}}$ with ' $x$ ' exactly resembles that of the saturation field vs composition ( $x$ ) (figures 9 and 10).

These results indicate that the saturation field, $H_{\mathrm{s}}$, in the series is determined by the saturation magnetization, $M_{\mathrm{s}}$, that in turn is decided by the composition ' $x$ '. From figures 4-8 it can be seen that the linear part in the graph can be employed to sense the magnetic fields and the maximum field that the film can sense is determined by the ' $x$ ' value.

\section{Conclusions}

Complex behaviour is observed in the field induced laser transmission through ferrofluid thin films belonging to the series, $\mathrm{Ni}_{x} \mathrm{Fe}_{1-x} \mathrm{Fe}_{2} \mathrm{O}_{4}$. The diffracted light intensity is increased by the application of the magnetic field while the intensity of the central spot decreases. The output intensity of the central spot decreases in a linear manner till saturation occurs in the cluster formation and this saturation field is dependent on the saturation magnetization $\left(M_{\mathrm{s}}\right)$ value of the ferrite powder precursors used for the ferrofluid synthesis.

\section{Acknowledgements}

(MRA) and (SSN) thank the Department of Science and Technology (File No-SP/S2/M-64/96 dated 22/04/2002) for necessary funding. (MRA) is also grateful to TWAS (File No-00-118 RG/PHYS/AS) for funding. The authors would like to thank Dr V P N Nampoori and Mr K P Unnikrishnan, International School of Photonics, CUSAT, Cochin, for fruitful discussions.

\section{References}

Berkovsky B M, Medvedev V S and Krakov M S 1993 Magnetic fluids; Engineering applications (Oxford: Oxford University Press) 
Chikazumi S, Taketomi S, Ukita M, Mizukami M, Miyajima H, Setogawa M and Kurihara Y 1987 J. Magn. \& Magn. Mater. 65245

Cullity B D 1978 Elements of X-ray diffraction (London: Addison Wesley Publishing Company) 2nd ed

Davies Helen W and Llewellian P 1979 J. Phys. D, Appl. Phys. 121357

Do Tengda and Luo Weili 1999 J. Appl. Phys. 855953

Eberberk Diatmar and Ahlers Heiko 1999 J. Magn. \& Magn. Mater. 192148

Hasmonay E and Depeyrot J 2000 J. Appl. Phys. 886628

Hong Chin-Yih 1999 J. Appl. Phys. 855962

Horng H E, Hong Chin-Yih, Yang S Y and Yang H C $2001 \mathrm{~J}$. Phys. \& Chem. Solids 621749

Ishi Yasushi 1998 J. Phys. Soc. Jap. 673050

JCPDS-ICDD C 1990 10-319

Kittel C 1971 Introduction to solid state physics (London: John Wiley) 4th ed

Luo Weili, Nagel Sydney R, Rosenbaum T F and Rosenweig R E 1991 Phys. Rev. Letts 672721
Malini K A, Sindhu S, Mohammed E M and Anantharaman M R 2001 J. Mater. Sci. 365551

Morosov K I, Phenichnikov A F, Raiker Yu L and Shilomis M I 1987 J. Magn. \& Magn. Mater. 65269

Rosenweig Ronald E 1985 Ferro hydrodynamics (Cambridge University Press)

Scholten P C 1980 IEEE Trans. Magn. 16221

Smit J and Wijn H P G 1959 Ferrites (Eindhovan: Philips Technical Library)

Spinu L, Srikanth H, Wiemwnn J A, Li S, Tang J and O'Conner C J 2000 IEEE Trans. Magn. 363062

Sutharia G M, Siblini A, Blanc-Mignon M F, Jorat L, Parekh K, Upadhyaya R V, Mehta R V and Noyel B 2001 J. Magn. \& Magn. Mater. 23490

Vasile Iusan Z and Aurora Gh Stanci 1994 IEEE Trans. Magn. 301104

Wu K T and Yao Y D 1999 J. Appl. Phys. 855959

Wu K T, Kuo P C, Yao Y D and Tsai E H 2001 IEEE Trans. Magn. 372651 